\title{
Basic Investigation of Vascular Interventional Radiology (IR) Using Large Rabbits
}

\author{
Norihisa NITTA $^{1)}$, Akinaga SONODA ${ }^{1)}$, Ayumi NITTA-SEKO ${ }^{1)}$, Shinichi OHTA ${ }^{1)}$, \\ Keiko TSUCHIYA $^{1)}$, Toyohiko TANAKA ${ }^{1)}$, Shuzo KANASAKI ${ }^{1)}$, \\ Kenichi MUKAISHO ${ }^{2)}$, Masashi TAKAHASHI ${ }^{1)}$, and Kiyoshi MURATA ${ }^{1)}$ \\ ${ }^{1)}$ Department of Radiology, Shiga University of Medical Science, Seta, Tsukinowa-cho, Otsu, \\ Shiga 520-2192 and ${ }^{2)}$ Department of Pathology, Shiga University of Medical Science, \\ Seta, Tsukinowa-cho, Otsu, Shiga 520-2192, Japan
}

\begin{abstract}
The purpose of this study was to determine the usefulness of large rabbits for basic vascular interventional radiology (IR) experiments. We used 5 Akita large rabbits (Akita) and 5 Japanese white rabbits (JW). We conducted measurements of vessel diameters such as the aorta, and the iliac, renal, superior mesenteric, celiac, and proper hepatic arteries, and of the growth rates of VX2 liver tumors. There were significant differences between Akita and JW in the diameters of the thoracic aorta, lower abdominal aorta, and celiac artery. In other blood vessels, no significant differences were found. There was no difference in the growth rates of the VX2 tumors between Akita and JW. The possibility that Akita large rabbits could be utilized for vascular IR was demonstrated.
\end{abstract}

Key words: angiography, large rabbit, VX2 liver tumor model

Rabbits have served as an experimental model for many basic studies in interventional radiology (IR). We have also utilized rabbits to study transcatheter arterial embolization [6]. However, ordinary rabbits have smallsized vessels, including the hepatic artery, and that limits the options in choosing vessels to be manipulated. Therefore, we conducted this study with large rabbits to determine their usefulness for basic vascular IR experiments.

All experiments were approved in advance by the Animal Experiment Ethical Committee of Shiga University of Medical Science. In all experiments, all rabbits were anesthetized with intramuscular injections of a mixture of ketamine hydrochloride $(25 \mathrm{mg} / \mathrm{kg}$, Ketalar 50; Sankyo Yell Yakuhin Co., Ltd.) and medetomidine hydrochloride $(0.1 \mathrm{mg} / \mathrm{kg}$, Domitor, Meiji Seika Co., Ltd., Tokyo, Japan).

In Experiment 1, measurement of vessel diameters, we used 5 Akita large rabbits (hereafter referred to as Akita) weighing between 6.34 and $7.30 \mathrm{~kg}$ (mean 6.93 $\mathrm{kg}$ ), aged 18-24 months, and 5 Japanese white rabbits (hereafter referred to as JW) weighing between 2.70 and $2.86 \mathrm{~kg}$ (mean $2.79 \mathrm{~kg}$ ), aged 18-24 months (Fig. 1). Vessel diameters were measured by a digital subtraction angiography (DSA) system. Measurements of the aorta, and the iliac, renal, celiac, superior mesenteric, and 


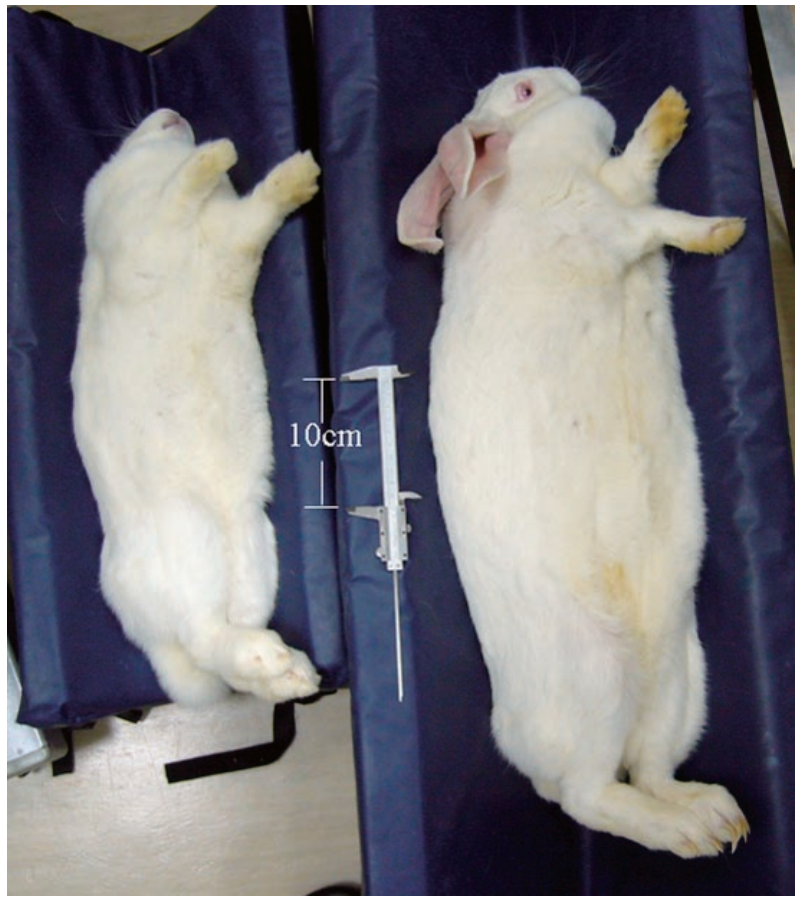

Fig. 1. Right: Akita large rabbit; Left: Japanese white rabbit. It can be clearly seen that Akita rabbits are larger than Japanese white rabbits.

proper hepatic arteries were made. The DSA system was manufactured by Shimadzu Co., and a C arm was employed for operative use. An image of a 5-cm metal scale was obtained for reference, and positioned in the same location on all of the DSA images of the target vessels. Diameters of the arteries were measured at the following levels: at the carina level for the thoracic aorta; at the level between the superior mesenteric and celiac arteries branched from the upper abdominal aorta; at the level just before the iliac artery branches to the lower abdominal aorta; at the level just before the internal and external iliac arteries branch to the left iliac artery; and at the level immediately after branching from the aorta to the celiac, superior mesenteric, and renal arteries, respectively. The diameter of the proper hepatic artery was measured at the level immediately after the gastroduodenal artery branch. For measurements of blood vessels, a 4-French sheath was inserted into the right femoral artery by the cut-down method. First, a 4-French pig-tail catheter (Clinical Supply Co., Ltd., Gifu, Japan) was inserted into the aortic arch and aortography was performed by manually injecting $5 \mathrm{ml}$ of $50 \%$ diluted
Iopamidol (Bayer Health Care Co., Ltd., Osaka, Japan). Subsequently, the celiac artery was selected using a 4-French cobra type catheter (Clinical Supply Co., Ltd., Gifu, Japan), and the proper hepatic artery was selected using a 2.1-Fr microcatheter (Sniper 2 selective type; Clinical Supply Co., Ltd., Gifu, Japan). Selection of proper hepatic arteries was conducted by two radiologists for all rabbits.

In Experiment 2, VX2 liver tumors were prepared and their growth rates were measured. VX2 tumor pieces measuring $2 \times 3 \times 3 \mathrm{~mm}$ were transplanted beneath the liver capsule in the same Akita and JW rabbits used in Experiment 1. Growth rates of the transplants were measured by studying changes in volume as measured by a horizontal open MRI system (0.3-Tesla coil; Hitachi Co., Ltd., Tokyo, Japan) on 2 and 3 weeks following transplantation. To obtain magnetic resonance (MR) images, a spin-echo pulse sequence T1-weighted image was used (repetition time (TR), $300 \mathrm{~ms}$; echo time (TE), $14 \mathrm{~ms}$; average, 20; field of view (FOV), $150 \mathrm{~mm}$; matrix size, $224 \times 192$; slice thickness, $3 \mathrm{~mm}$; and interslice gap, $0.5 \mathrm{~mm}$ ). Images were interpolated to a $256 \times 256$ matrix on a data server, saved as bitmap (BMP) files, and transferred to a personal computer. Liver tumor volumes were estimated using Photoshop 7.0.1 (Adobe Systems, Inc., San Jose, CA, USA) as follows. The tumors were manually outlined on each slice by two radiologists (N.N., A.S.) and after a consensus was reached, the total number of pixels was multiplied by $1.37 \mathrm{~mm}^{3}$. Relative tumor growth rates (total number of pixels at 3 weeks/total number of pixels at 2 weeks $\times 100)(\%)$ were calculated. Values of more than $100 \%$ signified an increase in tumor size, while values of less than $100 \%$ corresponded to a decrease.

After conducting MRI at 3 weeks after transplantation, all rabbits were sacrificed with overdose injections of pentobarbital (Nembutal, Dainippon Sumitomo Pharma Co., Ltd., Tokyo, Japan) to collect liver tumor specimens for pathologic analyses. They were fixed in 5\% formaldehyde and embedded in paraffin for $3-\mu \mathrm{m}$ transverse tissue section. Hematoxylin and eosin staining was performed and general morphology was evaluated using light microscopy.

All results are expressed as the mean \pm the standard deviation of the mean (SD). They were statistically 
Table 1. Measurement of aorta and arteries

\begin{tabular}{lccccccccccccccc}
\hline & Akita 1 & Akita & \multicolumn{1}{l}{ Akita 3} & Akita & Akita 5 & Mean & SD & JW 1 & JW 2 & JW 3 & JW 4 & JW 5 & Mean & SD & $P$ value \\
\hline TA & 5.6 & 5.9 & 6.4 & 5.8 & 5.9 & 5.92 & 0.29 & 5.3 & 4.9 & 5.9 & 5.4 & 5.3 & 5.36 & 0.36 & 0.02 \\
AA1 & 4.5 & 5.5 & 5.7 & 5.6 & 5.6 & 5.33 & 0.50 & 4.9 & 5.3 & 5.3 & 5.2 & 5.1 & 5.16 & 0.17 & 0.37 \\
AA2 & 3.0 & 4.4 & 4.9 & 4.8 & 4.5 & 4.32 & 0.77 & 2.4 & 3.0 & 3.0 & 2.6 & 2.6 & 2.72 & 0.27 & 0.00 \\
Lt IL & 2.7 & 2.0 & 2.7 & 2.3 & 2.2 & 2.38 & 0.31 & 2.3 & 2.3 & 1.9 & 2.2 & 2.1 & 2.16 & 0.17 & 0.20 \\
Rt Renal & 1.9 & 2.0 & 1.9 & 1.8 & 1.9 & 1.90 & 0.07 & 1.5 & 2.3 & 2.4 & 2.0 & 2.2 & 2.08 & 0.36 & 0.30 \\
Lt Renal & 1.9 & 1.5 & 1.5 & 1.7 & 1.8 & 1.68 & 0.18 & 1.5 & 1.7 & 2.3 & 1.8 & 1.6 & 1.78 & 0.31 & 0.55 \\
SMA & 2.7 & 2.2 & 2.7 & 2.5 & 2.6 & 2.54 & 0.21 & 2.3 & 2.7 & 2.4 & 2.4 & 2.5 & 2.46 & 0.15 & 0.50 \\
Celiac a & 2.3 & 2.4 & 2.7 & 2.6 & 2.5 & 2.50 & 0.16 & 2.1 & 2.3 & 2.3 & 2.1 & 2.2 & 2.20 & 0.10 & 0.00 \\
PHA & 0.87 & 0.84 & 1.01 & 0.93 & 0.90 & 0.91 & 0.065 & 0.85 & 0.91 & 0.94 & 0.88 & 0.90 & 0.896 & 0.034 & 0.68 \\
\hline
\end{tabular}

TA: thoracic aorta, AA1: upper abdominal aorta, AA2: lower abdominal aorta, IL: iliac artery, SMA: superior mesenteric artery, PHA: proper hepatic artery.

Table 2. Tumor growth rate

\begin{tabular}{|c|c|c|c|c|c|c|}
\hline Akita 1 & Akita 2 & Akita 3 & Akita4 & Akita 5 & Mean & SD \\
\hline 313.4 & 315.2 & 347.8 & 521.1 & 786.2 & 456.7 & 203.2 \\
\hline Japanese 1 & Japanese2 & Japanese 3 & Japanese 4 & Japanese 5 & Mean & SD \\
\hline 355.2 & 546 & 256.1 & 347.8 & 234.9 & 345 & 12.3 .0 \\
\hline
\end{tabular}

analyzed using Dr SPSS II for Windows standard version (SPSS Japan Inc., Tokyo, Japan). Diameter of blood vessels and relative tumor volume growth rates between Akita and JW were compared with Student's $t$-test. $P<0.05$ was considered statistically significant.

Mean vessel diameters \pm standard deviation (in $\mathrm{mm}$ ) in Akita and JW, respectively, were as follows: descending aorta, $5.92 \pm 0.29$ vs $5.36 \pm 0.36$; upper abdominal aorta, $5.33 \pm 0.50$ vs $5.16 \pm 0.17$; lower abdominal aorta, $4.32 \pm 0.77$ vs $2.72 \pm 0.27$; iliac artery, $2.38 \pm 0.31$ vs $2.16 \pm 0.17$; right and left renal arteries, $1.90 \pm 0.07$ vs $2.08 \pm 0.36$ and $1.68 \pm 0.18$ vs $1.78 \pm 0.31$; celiac artery, $2.50 \pm 0.16$ vs $2.20 \pm 0.10$; superior mesenteric artery, $2.54 \pm 0.21$ vs $2.46 \pm 0.15$; and proper hepatic artery, $0.910 \pm 0.065$ vs $0.896 \pm 0.034$ (Table 1). As summarized in Table 1, the aorta of Akita were 11\% larger aorta, celiac arteries $14 \%$ larger, and proper hepatic arteries $2 \%$ larger than those of their JW counterparts. There were significant differences between Akita and JW in the diameters of the thoracic aorta, lower abdominal aorta, and celiac artery. In other blood vessel diameters, no significant differences were found.

Concerning the selection of the proper hepatic artery for microcatheter manipulation, the 2 radiologists could select the proper hepatic artery without causing vasospasm in any of the 5 Akita, whereas they could only do so in 2 of the $5 \mathrm{JW}$. There was no difference in the growth rate of the VX2 tumors between Akita (456.7 \pm $203.2 \%)$ and JW (345.0 $\pm 123.0 \%)$ (Table 2). Both species showed lower T1-weighted signals in the transplanted tumors than in the hepatic parenchyma. In contrast-enhanced MR images, homogeneous enhancing effects were observed in the tumors at 2 weeks, but portions inside the tumors were poorly enhanced at 3 weeks, indicating necrosis of portions of the tumors (Figs. 2A-D). We also confirmed tumor properties in pathological examinations, the observations of which were the same in both Akita and JW.

Our attention was directed to large rabbits that were historically raised in the Senhoku area of Akita prefecture in Japan. These rabbits were produced as an improved large breed of Akita from JW through repeated crossing with New Zealand white and Flemish Giant rabbits. This study was undertaken to determine whether these large rabbits could be utilized for IR experiments. 

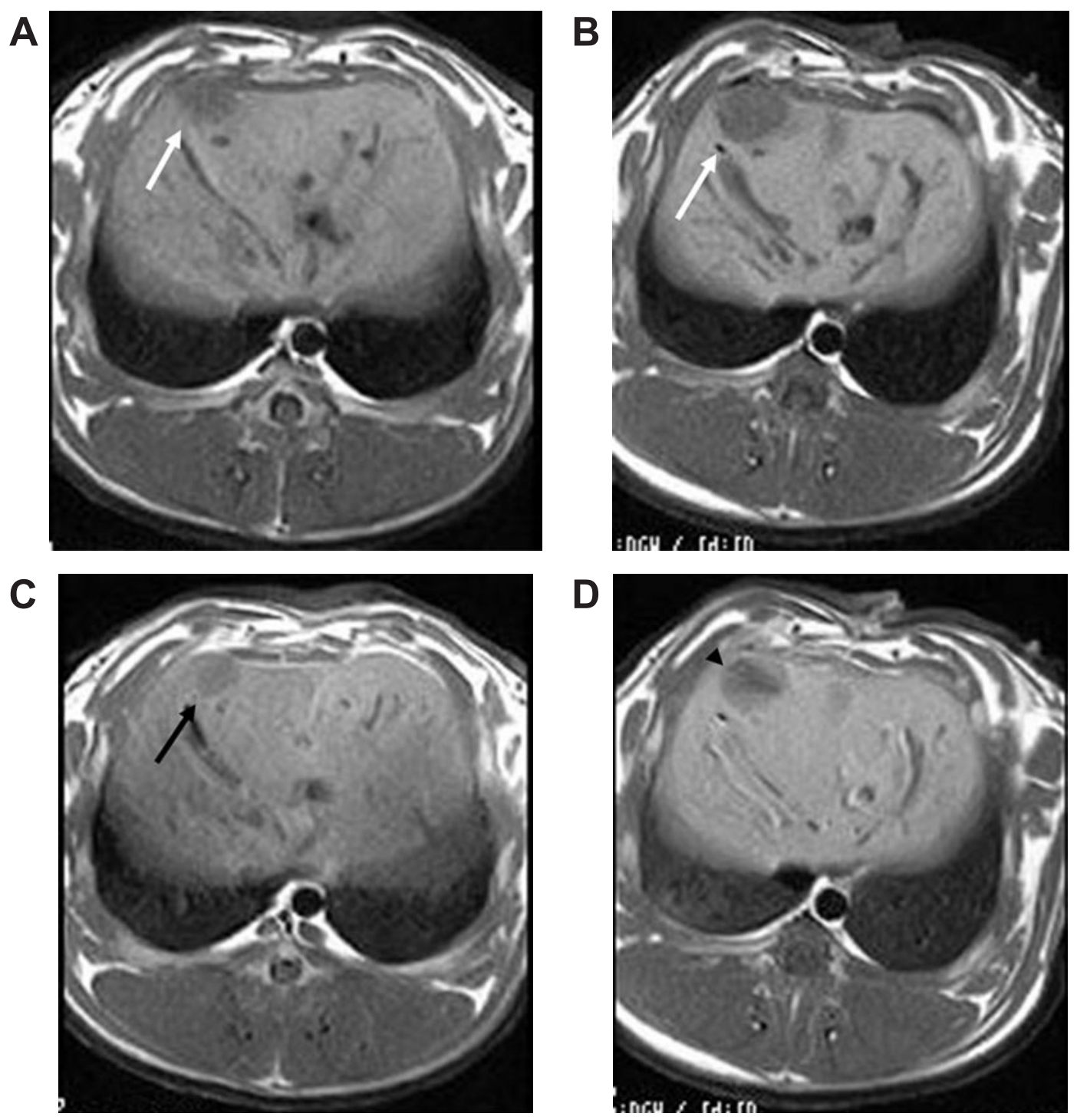

Fig. 2. A: T1-weighted image of a VX2 tumor two weeks after liver implantation. B: T1-weighted image of a VX2 tumor three weeks after liver implantation. C: Contrast-enhanced image of a VX2 tumor two weeks after liver implantation. D: Contrast-enhanced image of a VX2 tumor three weeks after liver implantation. The tumor sizes at three weeks following liver implantation were obviously larger than those at two weeks (white arrows). In contrast-enhanced images, practically homogeneous enhancing effects were observed in the tumors at two weeks (black arrow), but portions inside the tumors were poorly enhanced at three weeks (black arrowhead), indicating necrosis of portions of tumors which was confirmed by pathological examination.

Vessel diameters in Akita were generally about $10 \%$ larger than those of JW, except for the renal arteries. Our present results demonstrate that the rate of incidental vascular injuries occurring during manipulation of intravascular catheters can be reduced because Akita had vessels $10-15 \%$ larger in diameter and 20\% larger in area over their JW counterparts. Moreover, as liver tu- mor models using VX2 tumors, Akita rabbits did not differ with respect to tumor growth rate, MR images, or pathologic images from their JW counterparts. We thus conclude that experiments employing VX2 tumors are feasible with the use of Akita rabbits.

Rabbits are generally gentle, enabling their use for various types of experiments. For vascular IR experi- 
ments, small animals like mice or rats cannot be used because of their size limitations. When dogs or pigs are used for such experiments, several operators and assistants are needed. On the other hand, a single operator is sufficient for rabbits in most cases, which is a great advantage. The Akita used in the current study were as gentle as JW, facilitating the IR experiment which was conducted by a single operator. With the use of Akita, a variety of models are easily created, such as atherosclerosis models [5], liver tumor models using VX2 tumors [6], and indwelling reservoir models [7]. Hence, we speculate that basic IR experiments will be easier to conduct using Akita than JW.

Certain issues concerning the use of rabbits and whether or not therapeutic effects can be evaluated for endovascular surgery like tansarterial embolization must be addressed. In embolotherapy using JW, a microcatheter must be introduced into the proper hepatic artery. Such manipulation by itself often causes vascular spasms or at most vascular obstructions. In contrast, microcatheters can be easily introduced into the proper hepatic artery of Akita. From the present results, we consider it possible to evaluate true therapeutic effects of transarterial embolization (TAE) without problems of vascular spasms or other obstructions. However, angiographic findings showed that there was no significant difference in the diameters of the proper hepatic arteries of both rabbits. In the selection experiment of proper hepatic arteries by two radiologists, vasospasms were observed more frequently in JW than in Akita; however, we could be elaborate on this result. In the future, physiological, pharmacological, and pathological investigations of blood vessels in Akita will be necessary.

It is regretful that our large rabbits, as well as other large varieties bred outside Japan as mentioned above, are currently not available as specific pathogen free (SPF) animals except for the New Zealand White species [1]. Our species was found to be infected with Coccidium, Pasteurella, and Bordetella bronchiseptica. Such pathogenic microorganisms need to be eliminated in a specially-designed facility producing SPF rabbits. Akita are allowed to be bred only in facilities that are licensed to breed conventional animals. The current status of experimental animals calls for SPF rabbits, the production of which is now being studied in Japan; however, other varieties of large rabbits exist in other parts of the world [2-4]. Large rabbits are expected to become SPF in every part of the world and to contribute to basic experiments for further progress of IR.

In conclusion, the possibility that Akita large rabbits could be utilized for vascular IR was demonstrated. Breeding of large SPF rabbits is a current issue that needs to be resolved.

\section{References}

1. Dufty, J.H. 1976. Aust. Vet. J. 52: 355-361.

2. Fontanesi, L., Tazzoli, M., Beretti, F., and Russo, V. 2006. Anim. Genet. 37: 489-493.

3. Hong, M.K., Vossoughi, J., Haudenschild, C.C., Eong, S.C., Xurkerman, B.D., and Leon, M.B. 1995. Am. Heart J. 130: 758-764.

4. Nakamura, A., DeMaria, T.F., Lim, D.J., Arya, G., and van Blitterswijk, C. 1991. Ann. Otol. Rhinol. Laryngol. 100: 1024-1031.

5. Nitta, N., Seko, A., Sonoda, A., Tanaka, T., Takahashi, M., Murata, K., Takemura, S., Sakamoto, T., and Tabata, Y. 2008. Cardiovasc. Intervent. Radiol. 31: 359-366.

6. Ohta, S., Nitta, N., Sonoda, A., Seko, A., Tanaka, T., Takahashi, M., Takemura, S., Tabata, Y., and Murata, K. 2008. Eur. J. Radiol. (in press).

7. Seko, A., Nitta, N., Sonoda, A., Ohta, S., Takahashi, M., Murata, K., and Tabata, Y. 2009. AJR. Am. J. Roentgenol. 192: w306-310. 